Pacific Journal of Mathematic 


\title{
GROWTH TRANSFORMATIONS FOR FUNCTIONS ON MANIFOLDS
}

\author{
Leonard E. Baum and George R. Sell
}

In this paper we look at the problem of maximizing a function $P$ defined on a manifold $M$. Although we shall be primarily concerned with the case where $M$ is a certain polyhedron in a Euclidean space $R^{n}$ and $P$ is a polynomial with nonnegative coefficients defined on $R^{n}$, some of our results are valid in greater generality.

In $\S 2$ we describe the general behavior of a growth transformation of $P$ in the vicinity of a local extremum. These results are of a topological nature and can be thought of as a topological-dynamical description of growth transformations.

In $\S 3$ we turn our attention to a particular class of growth transformation which arise for polynomials with nonnegative coefficients. We shall prove the following result, which is the main theorem of this paper:

THeorem. Let $M \cup \partial M$ denote the manifold with boundary given by $x=\left(x_{i i}\right)$ where

$$
\left\{x_{i j}: x_{i j} \geqq 0 \text { and } \sum_{j=1}^{q_{i}} x_{i j}=1\right\}
$$

where $q_{1}, \cdots, q_{k}$ is a set of nonnegative integers. Let $P$ be a homogeneous polynomial in the variable $\left\{x_{i j}\right\}$, with nonnegative coefficients. Let $\mathscr{T}=\mathscr{T}_{P}: M \rightarrow M \cup \partial M$ defined by $y=\mathscr{T}_{P}(x)$ where

$$
y_{i j}=x_{i j} \frac{\partial P}{\partial x_{i j}}\left[\sum_{k=1}^{q_{i}} x_{i k} \frac{\partial P}{\partial x_{i k k}}\right]^{-1} .
$$

Then

$$
P(x) \leqq P\left(t \mathscr{T}_{P}(x)+(1-t) x\right), \quad(0 \leqq t \leqq 1, x \in M)
$$

The proof of this is based on a suitable modification of an argument of L. E. Baum and J. A. Eagon, cf., [1].

We also study the problem of extending the mapping $\mathscr{T}_{P}$ to the boundary $\partial M$ in such a way that it is continuous. These results are stated in Theorem 7. It is a consequence of this that $\mathscr{T}_{P}$ maps neighborhoods of a local maximum into themselves even if the maximum is on the boundary.

In $\S 5$ we examine other growth transformations that are related 
to the mapping $\mathscr{T}_{P}$. By using an argument suggested by Professor O. Rothaus we are able to extend the theorem stated above to arbitrary (nonhomogeneous) polynomials with positive coefficients.

2. Growth transformations. In this section we shall investigate the behavior of a growth transformation in the vicinity of an isolated maximum.

Definition. Let $P$ be a continuous function defined on a $C^{\infty}$ manifold $M$. We say that a continuous mapping $\sigma: M \rightarrow M$ is a growth transformation (for $P$ ) if

$$
P(x) \leqq P(\sigma(x)), \quad(x \in M) .
$$

If $P$ is a $C^{2}$-function we say that $\sigma$ is a proper growth transformation (for $P$ ) if (2) holds and

$$
P(x)=P(\sigma(x)) \text { implies that } x \text { is a critical point of } P,
$$

which means that grad $P=0$ at $x$. A growth transformation $\sigma$ is said to increase $P$ homotopically if there exists a continuous mapping

$$
S_{t}(x):[0,1] \times M \longrightarrow M
$$

such that

(4) (ii) $S_{1}(x)=\sigma(x)$

(iii) For each $t, 0 \leqq t \leqq 1, S_{t}$ is a growth transformation for $P$.

A continuous function $P$ is said to have a local maximum at $q$ if there is a neighborhood $V$ of $q$ with

$$
P(x) \leqq P(q)
$$

for all $x$ in $V$. If $P$ is a $C^{2}$-function, the point $q$ is called an isolated maximum if it is a local maximum of $P$ and an isolated critical point.

We will describe the asymptotic behavior of the iterates of a growth transformation. Let $\sigma$ be a growth transformation for $P$ and define the fixed value set $F_{P \sigma}$ by

$$
F_{P \sigma}=\{x \in M: P(x)=P(\sigma(x))\} .
$$

We say that a set $K \subset M$ is invariant if $\sigma^{n}(x) \in K$ for $n \geqq 1$, whenever $x \in K$.

Theorem 1. Let $P$ be a real-valued continuous function on a $C^{\infty}$-manifold $M$ and let $\sigma: M \rightarrow M$ be a growth transformation for 
$P$. Let $K$ be the largest invariant subset of the fixed value set $F_{P \sigma}$ given by (5). If a point $x$ in $M$ has the property that $\sigma^{n}(x)$ remains in a compact for $n \geqq 1$, then

$$
\sigma^{n}(x) \longrightarrow K \text { as } n \longrightarrow \infty \text {. }
$$

Proof. Let $x \in M$ be a point with the property that $\{x, \sigma(x)$, $\left.\sigma^{2}(x), \cdots\right\}$ lies in a compact set in $M$. Since $P\left(\sigma^{n}(x)\right)$ is increasing in $n$, it follows that $\lim _{n \rightarrow \infty} P\left(\sigma^{n}(x)\right)$ exists, say that

$$
P\left(\sigma^{n}(x)\right) \longrightarrow \alpha \text { as } n \longrightarrow \infty \text {. }
$$

Now let $y \in M$ be a limit point of a subsequence $\left\{\sigma^{n i}(x)\right\}$. Then by the continuity of $P$ we have

$$
P\left(\sigma^{n}(x)\right) \longrightarrow P(y)=\alpha .
$$

However $\sigma^{n_{i+1}}(x) \rightarrow \sigma(y)$ and in general $\sigma^{n_{i+k}}(x) \rightarrow \sigma^{k}(y)$. From this it follows that $P\left(\sigma^{k}(y)\right)=\alpha, k=0,1, \cdots$, in other words, $y \in K$, which completes the proof of Theorem 1 .

By demanding that the growth transformation $\sigma$ leave the local maxima of $P$ fixed, we are able to assert something about the behavior of $\sigma$ in the vicinity of a local maximum.

THEOREM 2. Let $P$ be a real-valued $C^{2}$-function on a $C^{\infty}$-manifold $M$ and let $\sigma: M \rightarrow M$ be a growth transformation for $P$. Assume that every local maximum of $P$ is a fixed point for $\sigma$. Then for every local maximum $q$ of $P$ there is a neighborhood $V$ with $\sigma(V) \subset V$. If, in addition, $\sigma$ is a proper growth transformation and $q$ is an isolated maximum than $V$ can be chosen so that

$$
\sigma^{n}(x) \longrightarrow q \text { as } n \longrightarrow \infty
$$

for every $x \in V$.

Proof. Let $q$ be a local maximum of $P$ and let $V$ be the connected component of

$$
\{x: P(x)>P(q)-\eta\}
$$

that contains $q$, where $\eta>0$ is fixed. Furthermore, we can choose $\eta$ so that $\bar{V}$, the closure of $V$, is compact. Now define

$$
\begin{aligned}
& A=\{x \in V: \sigma(x) \in \bar{V}\} \\
& B=\{x \in V: \sigma(x) \notin \bar{V}\} .
\end{aligned}
$$

It is clear that $A \cap B=\varnothing$ and $V=A \cup B$. Furthermore, $B$ is an open set since $\sigma$ is continuous. The set $A$ can be written as 


$$
A=\{x \in V: \sigma(x) \in V\}
$$

since $\sigma$ is a growth transformation, that is the set

$$
\{x \in V: \sigma(x) \in \bar{V}-V\}=\{x \in V: P(x)=P(q)-\eta\}
$$

is empty. It follows then that $A$ is an open set. Since $V$ is connected one of the sets $A$ or $B$ must be empty. However, $\sigma(q)=q$, therefore $B=\varnothing$. Hence $V=A$ and $\sigma(V) \subset V$. (It also follows that $\sigma(\bar{V}) \subset \bar{V}$.)

If $q$ is a maximum, that is an isolated critical point, then $\eta$ can be chosen so that $\bar{V}$ contains no critical point of $P$ other than $q$. If $K$ is the largest invariant subset of $F_{P o}$ which is given by (5), then $K \cap V=\{q\}$. Hence by Theorem 1, if $x \in V$, then $\sigma^{n}(x) \rightarrow q$ as $n \rightarrow \infty$.

A conclusion similar to that of Theorem 2 is possible under a slightly different hypothesis.

Theorem 3. Let $P$ be a real-valued continuous function defined on a $C^{\infty}$-manifold $M$ and let $\sigma: M \rightarrow M$ be a growth transformation for $P$. Assume that $\sigma$ increases $P$ homotopically and let $S_{t}(x)$ satisfy (4). Then for every local maximum $q$ of $P$ there is a neighborhood $V$ with $S_{t}(V) \subset V, 0 \leqq t \leqq 1$. If, in addition, $\sigma$ is a proper growth transformation and $q$ is an isolated maximum, then $V$ can be chosen so that for every $x \in V$

$$
\sigma^{n}(x) \longrightarrow q \text { as } n \longrightarrow \infty,
$$

in particular $\sigma(q)=q$.

Proof. Let $q \in M$ be a local maximum of $P$ and let $V$ be the component of $\{x: P(x)>P(q)-\eta\}$ that contains $q$, where $\eta>0$ is fixed. We also choose $\eta$ so that $\bar{V}$ is compact. Now fix $x$ in $V$. Then

$$
P(q)-\eta<P(x) \leqq P\left(S_{t}(x)\right), \quad 0 \leqq t \leqq 1 .
$$

Let $G=\left\{S_{t}(x): 0 \leqq t \leqq 1\right\}$, then $G$ is a connected set since $S_{t}(x)$ is. continuous in $t$. By (6) we see that the set

$$
\left\{S_{t}(x) \in \bar{V}-V\right\}=\left\{S_{t}(x): P\left(S_{t}(x)\right)=P(q)-\eta\right\}
$$

is empty. Thus, $G=(G \cap V) \cup(G \cap(M-\bar{V}))$. Since $G$ is connected one of the sets $G \cap V$ or $G \cap(M-\bar{V})$ is empty. However, $x \in G \cap V$. Hence $G \subset V$, that is $S_{t}(V) \subset V$ for $0 \leqq t \leqq 1$. The remainder of the proof follows that of Theorem 2 .

In Theorems 2 and 3 one is able to assert that the neighborhood. $V$ is a disk provided the function $P$ is a $C^{2}$-function, cf. [4]. Also, 
these results are really "local" results, so they are still valid even if $\sigma$ and $P$ are defined only in a neighborhood of the maximum $q$. And, finally, they have obvious extensions to manifolds with boundary. Another refinement of Theorem 3, is the following.

THEOREM 4. Let $P$ be a real-valued continuous function defined on a $C^{\infty}$-manifold $M$ and let $\sigma: M \rightarrow M$ be a growth transformation that increases $P$ homotopically. If $q$ is an isolated maximum of $P$, then $\sigma(q)=q$.

Proof. Let $V_{\eta}$ be the component of $\{x: P(x)>P(q)-\eta\}$ that contains $q$. It was shown in the proof of Theorem 3 that $S_{t}\left(V_{\eta}\right) \subseteq V_{\eta}$. By letting $\eta \rightarrow 0$ we conclude that $\sigma(q)=q$.

The next result seems rather interesting. It asserts that, under appropriate conditions, every growth transformation increases $P$ homotopically in a neighborhood of an isolated maximum.

THEOREM 5. Let $P$ be a real-valued $C^{2}$-function on a $C^{\infty}$-manifold $M$ and let $\sigma: M \rightarrow M$ be a growth transformation. Assume that every isolated maximum of $P$ is a fixed point of $\sigma$. Then for every isolated maximum $q$ there is a neighborhood $V$ such that $\sigma$ increases $P$ homotopically in $V$.

Proof. Let $\eta>0$ be chosen so that the component $V=V_{\eta}$ of the set $\{x: P(x)>P(q)-\eta\}$ that contains $q$ has the property that $\bar{V}$ is compact and $\bar{V}$ contains no critical points of $P$ other than $q$. Now consider the differential equation

$$
x^{\prime}=-\operatorname{grad} P
$$

in $V_{\eta}$, and let $\varphi(x, t)$ denote the solution that satisfies $\varphi(x, 0)=x$. It is easy to show that for $x \in V_{\eta}, \varphi(x, t) \in V_{\eta}$, for $t \geqq 0$, and $\varphi(x, t) \rightarrow q$ as $t \rightarrow \infty$. Now choose $\varepsilon, 0<\varepsilon<\eta$, so that, in the local coordinate system at $q$, the convex hull of $V_{\varepsilon}$ lies in $V_{\eta}$. We now define a mapping $h: V_{\eta} \rightarrow V_{\varepsilon}$ by

$$
\begin{aligned}
& h(x)=x, \quad \text { if } \quad x \in V_{\varepsilon} \\
& h(x)=\varphi\left(x, T_{x}\right), \text { if } x \in V_{\eta}-V_{\varepsilon},
\end{aligned}
$$

where $\varphi\left(x, T_{x}\right)$ is the first point at which the trajectory $\varphi(x, t)$ meets $V_{\varepsilon}$. Since the level surface $P(x)=P(q)-\varepsilon$ is transverse to the flow $\varphi(x, t)$, it follows that $h$ is continuous. Also the mapping

$$
g: V_{\eta} \times[0,1] \rightarrow V_{r}
$$


given by

$$
g(x, \tau)=\tau h(\sigma(x))+(1-\tau) h(x)
$$

is continuous. Now define $S_{\tau}(x), 0 \leqq \tau \leqq 1$ and $x \in V_{\eta}$, to be that point in $V_{\eta}$ on the trajectory $\varphi(g(x, \tau), t)$ that satisfies

$$
P\left(S_{\tau}(x)\right)=\tau P(\sigma(x))+(1-\tau) P(x) .
$$

It is clear that $S_{\tau}(x)$ is continuous, and it is easy to verify that $S_{\tau}(x)$ satisfies (4). This completes the proof of the theorem.

It is apparent from the proofs that if $\sigma$ is a proper growth transformation then the region of attraction of an isolated maximum $q$ is "large". More precisely, let $\eta>0$ and define $V_{\eta}$ to be the component of $\{x: P(x)>P(q)-\eta\}$ that contains $q$. We have seen that the region of attraction for $q$ will contain every $V_{\eta}$ that has the property that the closure $\bar{V}_{\eta}$ is compact and contains only one critical point of $P$, which must necessarily be the point $q$. If we let $\eta_{0}>0$ be the first real number for which $\bar{V}_{\eta_{0}}$ contains more than one critical point of $P$ then since

$$
V_{\eta_{0}}=\bigcup_{\eta<\eta_{0}} V_{\eta},
$$

this implies that the region of attraction always contains $V_{\eta_{0}}$. It should be emphasized that $V_{\eta_{0}}$ depends only on the function $P$ and not on the growth transformation $\sigma$.

3. Homogeneous polynomials and the transformation $\mathscr{T}_{p}$. Let $q_{1}, \cdots, q_{k}$ be a set of nonnegative integers with $\Sigma_{i} q_{i}=n$. Let $M \cup \partial M$ denote the set of all vectors

$$
\left\{x=\left(x_{i j}\right), i=1, \cdots, k, j=1, \cdots, q_{i}\right\}
$$

such that

$$
x_{i j} \geqq 0 \quad \text { and } \quad \sum_{j=1}^{q_{i}} x_{i j}=1 .
$$

The set $M \cup \partial M$ is a polyhedron in $R^{n}$. We shall let $M$ denote the interior of the polyhedron, that is

$$
M=\left\{\left(x_{i j}\right) \in M \cup \partial M: x_{i j}>0\right\}
$$

and $\partial M$ is the boundary. The space $M$ is a manifold of dimension $n-k$.

Let $P: R^{n} \rightarrow R$ be a homogeneous polynomial in the variables $\left(x_{i j}\right)$ with positive coefficients. We define a mapping

$$
\mathscr{T}=\mathscr{T}_{P}: M \rightarrow M \cup \partial M
$$


by $y=\mathscr{T}_{P}(x)$ where

$$
y_{i j}=\mathscr{T}_{P}(x)_{i j}=x_{i j} \frac{\partial P}{\partial x_{i j}}\left[\sum_{k=1}^{q_{i}} x_{i k} \frac{\partial P}{\partial x_{i k}}\right]^{-1} .
$$

Note that the range of $\mathscr{T}$ is contained in $M$ unless $P$ does not depend on one of the variables $x_{i j}$.

In [1] it was shown that $P(x) \leqq P(\mathscr{T}(x))$ for all $x \in M$ and equality held if and only if $\mathscr{T}(x)=x$. In other words, the transformation $\mathscr{T}_{P}$ is a growth transformation for $P$. We now can assert a stronger result.

THEOREM 6. The transformation $\mathscr{T}_{P}$ increases $P$ homotopically. More precisely, if $P$ is a homogeneous polynomial in $\left(x_{i j}\right)$ with positive coefficients and $\mathscr{T}_{P}=\mathscr{T}$ is given by (7), then

$$
P(x) \leqq P(t \mathscr{T}(x)+(1-t) x), \quad(x \in M, 0<t \leqq 1) .
$$

Moreover, equality holds in (8) if and only if $\mathscr{T}(x)=x$.

Note that the transformation $\mathscr{T}_{P}$ is determined by the first derivatives of $P$ only. In a sense it is similar to moving in the "gradient direction", which also depends on only the first derivatives of $P$. While moving in the gradient direction will increase the value of $P$, this is valid only for small steps, and there is no way-without considering second derivatives-for determining the size of the step. On the other hand, the size of the step is completely determined by the first derivatives above for the transformation $\mathscr{T}_{P}$.

Proof. One can write $P(x)=\Sigma_{\alpha} C_{\alpha} m_{\alpha}(x)$ where the coefficients $C_{\alpha}$ are positive and $m_{\alpha}(x)$ is a monomial of degree $d$, that is

$$
m_{\alpha}(x)=\prod_{i, j} x_{i j}^{\alpha_{i j}}
$$

where the $\alpha_{i j}$ are nonnegative integers with $\Sigma_{i}{ }_{j} \alpha_{i j}=d$. We shall let $\alpha=\left(\alpha_{i j}\right)$ be the index set for the summation defining $P$. We note now a few identities which will be needed later.

$$
\begin{gathered}
P(x)=\frac{1}{d} \sum_{i, j} x_{i j} \frac{\partial P}{\partial x_{i j}} \\
\sum_{\alpha} \alpha_{i j} C_{\alpha} m_{\alpha}(x)=x_{i j} \frac{\partial P}{\partial x_{i j}} \\
\frac{m_{\alpha}(x)}{m_{\alpha}(y)}=m_{\alpha}\left(\frac{x}{y}\right)
\end{gathered}
$$

where 


$$
\frac{x}{y}=\left(\frac{x_{i j}}{y_{i j}}\right)
$$

By the inequality of geometric and arithmetic means [3,p. 16] we get

$$
m_{\alpha}(x)^{1 / d} \leqq \frac{1}{d} \sum_{i, j} \alpha_{i j} x_{i j}
$$

Let $t, 0 \leqq t \leqq 1$, be fixed and let $y=t \mathscr{S}^{-}(x)+(1-t) x$. Also define $Q_{\alpha}$ by

$$
P(x)=\sum_{\alpha}\left\{C_{\alpha} m_{\alpha}(y)\right\}^{1 / d+1} Q_{\alpha}
$$

By applying Hölder's inequality [3, p. 21] to (11) we get

$$
P(x) \leqq P(y)^{1 / d+1}\left(\sum_{\alpha} Q_{\alpha}^{d+1 / d}\right)^{d /(d+1)}
$$

Now

$$
\begin{aligned}
\sum_{\alpha} Q_{\alpha}^{d+1 / d} & =\sum_{\alpha} C_{\alpha} m_{\alpha}(x)\left[\frac{m_{\alpha}(x)}{m_{\alpha}(y)}\right]^{1 / d} \\
& =\sum_{\alpha} C_{\alpha} m_{\alpha}(x)\left[m_{\alpha}\left(\frac{x}{y}\right)\right]^{1 / d} \\
& \leqq \frac{1}{d} \sum_{\alpha} C_{\alpha} m_{\alpha}(x) \sum_{i, j} \alpha_{i j}\left(\frac{x_{i j}}{y_{i j}}\right)
\end{aligned}
$$

where we apply (10) in the last step.

By substituting for $y_{i j}$ in (13) we get

$$
\sum_{\alpha} Q_{\alpha}^{(d+1) / d} \leqq \frac{1}{d} \sum_{\alpha} C_{\alpha} m_{\alpha}(x) \sum_{i} \sum_{j} \alpha_{i j}\left[\frac{\sum_{k} x_{i k} P_{i k}}{t P_{i j}+(1-t) \Sigma_{k} x_{i k} P_{i k}}\right]
$$

where

$$
P_{i j}=\frac{\partial P}{\partial x_{i j}}
$$

Now by interchanging the order of summation and using (9) we get

$$
\sum_{\alpha} Q_{\alpha}{ }^{(d+1) / d} \leqq \frac{1}{d} \sum_{i}\left(\sum_{k} x_{i k} P_{i k}\right)\left[\sum_{j} \frac{x_{i j} P_{i j}}{t P_{i j}+(1-t) \Sigma_{k} x_{i k} P_{i k}}\right] .
$$

However, by Lemma 1 (see below) with $a_{j}=x_{i j} P_{i j}, b_{j}=t P_{i j}$ and $c_{j}=(1-t) \Sigma_{k} x_{i k} P_{i k}$, we see that the quantity in the brackets in (14) is bounded by 1 for $0<t<1$, and by continuity it is bounded for $0 \leqq t \leqq 1$. Hence (14) becomes 


$$
\sum_{\alpha} Q_{\alpha}^{(d+1) / d} \leqq \frac{1}{d} \sum_{i} \sum_{k} x_{i k} P_{i k}=P(x)
$$

By putting this into (12) we get

$$
P(x) \leqq P(y)^{1 /(d+1)} P(x)^{d /(d+1)},
$$

which implies that $P(x) \leqq P(y)$, and this completes the proof of the theorem.

Lemma 1. Let $a_{j}, b_{j}, c_{j}, j=1, \cdots, n$, be positive numbers with $\sum_{j=1}^{n} a_{j} / b_{j} \leqq 1 / P$ and $\sum_{j=1}^{n} a_{j} / c_{j} \leqq 1 / Q$, then $\sum_{j=1}^{n} a_{j} /\left(b_{j}+c_{i}\right) \leqq 1 /(P+Q)$.

The proof of this is a straightforward induction argument and we will omit the details.

The following consequence of Theorems 3 and 6 asserts that the mapping $\mathscr{T}_{P}$ cannot leave a "local hill". Furthermore, we are able to conclude something about the region of attraction for an isolated local maximum of $P$.

COROLlary. Let $P$ be a homogeneous polynomial in the variables $\left(x_{i j}\right)$ with positive coefficients and let $q \in M$ be an isolated local maximum of $P$. Then there exists a neighborhood $V$ of $q$ such that $\mathscr{T}(V) \subset V$ and for every $x \in V$

$$
\mathscr{T}^{n}(x) \longrightarrow q \text { as } n \longrightarrow \infty \text {. }
$$

Observe that this corollary can also be obtained from Theorem 2 since $\mathscr{T}$ is a proper growth transformation and $\mathscr{T}(x)=x$ if and only if $x$ is a critical point of $P$.

The transformation $\mathscr{T}_{P}$ can, in a limited sense, be extended to the boundary $\partial M$

$$
\partial M=\bigcup_{i j} N_{i j} \cup \partial N_{i j}
$$

where each $N_{i j} \cup \partial N_{i j}$ is a polyhedron defined by

$$
N_{i j} \cup \partial N_{i j}=\left\{x \in M \cup \partial M: x_{i j}=0\right\} .
$$

Following our original convention, we shall let $N_{i j}$ denote the interior of $N_{i j} \cup \partial N_{i j}$ and $\partial N_{i j}$ the boundary.

THEOREM 7. (A) The transformation $\mathscr{T}_{P}$ on $M$ can be extended to be continuous, and in fact $C^{\infty}$, on

$$
\bigcup_{i j} N_{i j} \cup M \text {. }
$$

(B) $\mathscr{T}_{P}$ can also be continuously extended to any isolated local 
maximum $q$ of $P$ on $\partial M$ by the definition. $\mathscr{T}_{P}(q)=q$.

(C) The extended transformation $\mathscr{T}_{P}$ still obeys the inequality

$$
P(x) \leqq P\left(t \mathscr{T}_{P}(x)+(1-t) x\right), \quad(0 \leqq t \leqq 1) .
$$

The proof of this theorem and the following corollaries will be given at the end of the paper.

In an example below we will show that in general $\mathscr{T}_{P}$ cannot be continuously extended to all of $\partial N_{i j}$. This occurs when a saddle point of $P$ lies on $\partial N_{i j}$.

Note that if a local maximum $q$ lies on $\partial N_{i j}$, then statement (B) above does not assert that $\mathscr{T}$ can be extended to a neighborhood of $q$ in $M \cup \partial M$.

CoRollary 1 (A). Let $P$ be a homogeneous polynomial in the variables $\left(x_{i j}\right)$ with positive coefficients and let $q \in \bigcup_{i j} N_{i j}$ be an isolated maximum of $P$ on the boundary $\partial M$. Then there exists a neighborhood $V$ of $q$, such that $\mathscr{T}(V) \subset V$, and for every $x \in V$

$$
\mathscr{T}^{n}(x) \longrightarrow q \text { as } n \longrightarrow \infty \text {. }
$$

(B) If $q \in \bigcup_{i j} \partial N_{i j}$ is an isolated maximum of $P$, then there is a neighborhood $V$ of $q$ in $M \cup \partial M$ such that for every $x \in V \cap\left(M \cup \cup_{i j} N_{i j}\right)$

$$
\mathscr{T}^{n}(x) \longrightarrow q \text { as } n \longrightarrow \infty \text {. }
$$

Corollary 2. Let $q \in N_{i j}$ be an isolated local maximum of $P$ and let $x \in M \cup \partial M$ a point in the domain of attraction of $q$. Let $y^{n}=\mathscr{T}_{n}(x)$ have coordinates $\left(y_{r s}^{n}\right)$. Then $y_{i j}^{n} \rightarrow 0$ as $n \rightarrow \infty$. Furthermore, if $y_{i j}^{n}$ is sufficiently small, say that $y_{i j}^{n}<\delta$, then we can replace $y^{n}$ with $\widetilde{y}$ where $\widetilde{y}_{i j}=0,\left|\widehat{y}_{r s}-y_{r s}^{n}\right| \leqq \delta$ and $\mathscr{T}^{k}(\widetilde{y}) \rightarrow q$ as $k \rightarrow \infty$.

That is we can set the $i j$ component of $y^{n}$ equal to zero without destroying the convergence property. It should be noted that we do assume $q$ to be an isolated maximum of $P$ in $M \cup \partial M$. If $q$ is only an isolated maximum on $\partial M$ and not on $M \cup \partial M$, then it will still be a critical point of $P$, but it now will act as a saddle point in the discrete flow induced by $\mathscr{T}$. A simple example of this phenomenon is given by the polynomial

$$
P\left(x_{1}, x_{2}, y_{1}, y_{2}\right)=x_{1} x_{2}+y_{1} y_{2}
$$

on

$$
M \cup \partial M=\left\{\left(x_{1}, x_{2}, y_{1}, y_{2}\right): x_{i} \geqq 0, y_{i} \geqq 0, i=1,2, x_{1}+x_{2}=1, y_{1}+y_{2}=1\right\} .
$$


The points $\left(\frac{1}{2}, \frac{1}{2}, 0,1\right),\left(\frac{1}{2}, \frac{1}{2}, 1,0\right),\left(0,1, \frac{1}{2}, \frac{1}{2}\right)$ and $\left(1,0, \frac{1}{2}, \frac{1}{2}\right)$ are saddle points for $\mathscr{T}$ and they are local extremum for $P$ on $\partial M$, but are not extremum on $M \cup \partial M$.

4. An illustrative example. Let $M \cup \partial M$ be the subset of $R^{3}$ given by

$$
\left\{\left(x_{1}, x_{2}, x_{3}\right): x_{i} \geqq 0 \quad \text { and } \Sigma x_{i}=1\right\}
$$

and let $P(x)=x_{1}^{2}+4 x_{2} x_{3}$. Then on $M, \mathscr{T}(x)$ is given by

$$
\mathscr{T}:\left(x_{1}, x_{2}, x_{3}\right) \longrightarrow\left(\frac{x_{1}^{2}}{P}, \frac{2 x_{2} x_{3}}{P}, \frac{2 x_{2} x_{3}}{P}\right) .
$$

The range of $\mathscr{T}$ is then contained in the set

$$
\left\{\left(x_{1}, x_{2}, x_{3}\right) \in M \cup \partial M: x_{2}=x_{3}\right\}
$$

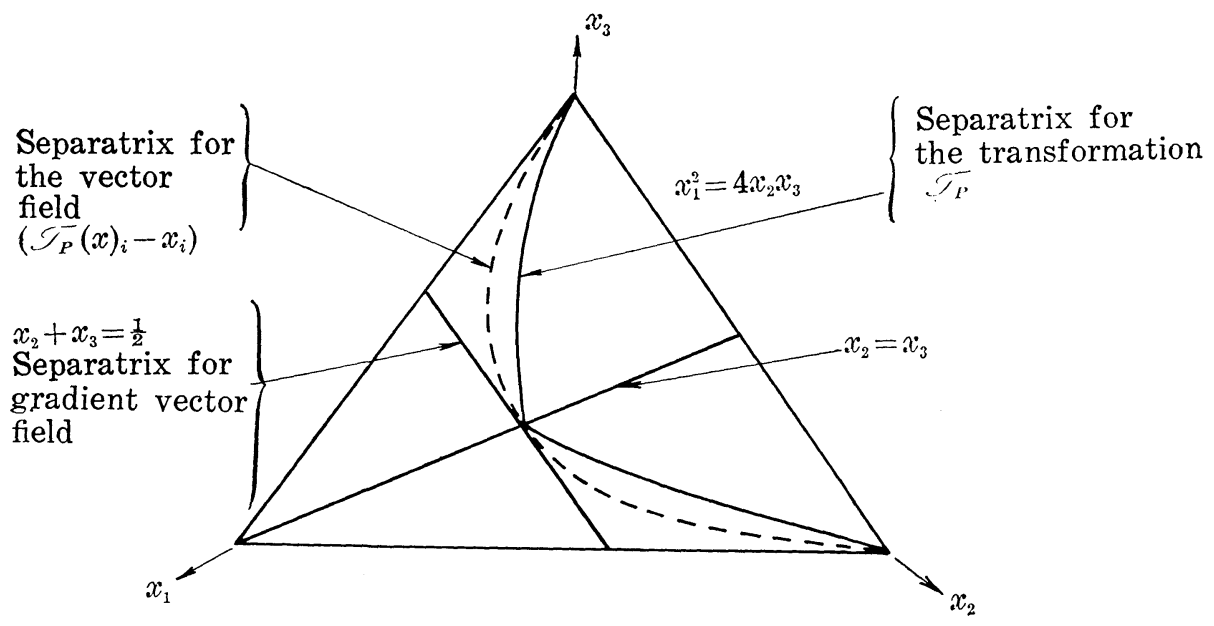

Figure 1.

The critical points of $P$ on $M \cup \partial M$ are:

$$
(1,0,0),(0,1,0),(0,0,1),\left(0, \frac{1}{2}, \frac{1}{2}\right),\left(\frac{1}{2}, \frac{1}{4}, \frac{1}{4}\right) \text {. }
$$

The points $(1,0,0)$ and $\left(0, \frac{1}{2}, \frac{1}{2}\right)$ are local maxima, $(0,1,0)$ and $(0,0,1)$ are local minima, and $\left(\frac{1}{2}, \frac{1}{4}, \frac{1}{4}\right)$ is a saddle point. The set of points

$$
\left\{\left(x_{1}, x_{2}, x_{3}\right) \in M: x_{1}^{2}=4 x_{2} x_{3}\right\}
$$

is mapped onto $\left(\frac{1}{2}, \frac{1}{4}, \frac{1}{4}\right)$ by $\mathscr{T}$. This forms a separatrix for the domains of attraction of $(1,0,0)$ and $\left(0, \frac{1}{2}, \frac{1}{2}\right)$. If $x_{1}^{2}>4 x_{2} x_{3}$, then $\mathscr{T}^{n}(x) \rightarrow\left(0, \frac{1}{2}, \frac{1}{2}\right)$, and if $x_{1}^{2}<4 x_{2} x_{3}$ then $\mathscr{T}_{n}(x) \rightarrow(1,0,0)$.

By taking limits from the inside, as indicated in Theorem $7, \mathscr{T}$ can be extended to all boundary points other than $(0,1,0)$ and $(0,0,1)$. ( $\mathscr{T}$ is defined and continuous even at the corner $(1,0,0)$ in this case.) 
The corners $(0,1,0)$ and $(0,0,1)$ are points of discontinuity of $\mathscr{T}$ since there are points arbitrarily close to either that are mapped near $(1,0,0)$ and other points that are mapped near $\left(0, \frac{1}{2}, \frac{1}{2}\right)$.

Finally let us show that the function of $t P(t \mathscr{T}(x)+(1-t) x)$ may fail to be monotone in $t$. We use this example, which is due to Blakely [2]. Let $x_{0}=(4 / 16,1 / 16,11 / 16)$

$$
\mathscr{T}(4 / 16,1 / 16,11 / 16)=(8 / 30,11 / 30,11 / 30) \text {. }
$$

Now

$$
\left.\frac{d}{d t} P(t \mathscr{T}(x)+(1-t) x)\right|_{t=1}=\left.\operatorname{grad} P\right|_{t=1} \cdot(\mathscr{T}(x)-x)
$$

and by a direct computation

$$
\left.\operatorname{grad} P\right|_{t=1} \cdot\left(\mathscr{T}\left(x_{0}\right)-x_{0}\right)<0 .
$$

Hence $P\left(t \mathscr{T}\left(x_{0}\right)+(1-t) x_{0}\right)$ is not monotone.

5. Growth transformations related to $\mathscr{T}_{P}$. Let us, for the moment, consider a special case of the polyhedron $M \cup \partial M$ discussed in $\S 3$, namely where $k=1$. Then $M \cup \partial M$ is the set of vectors $x=\left(x_{j}\right), j=1, \cdots, n$ such that $x_{j} \geqq 0$ and $\sum_{j=1}^{n} x_{j}=1$. In Theorem 3 , when we were studying the behavior of the transformation $\mathscr{T}_{P}$ generated by a homogeneous polynomial $P$, we were only interested in the behavior of $P$ on the polyhedron $M \cup \partial M$. Even though $P$ is fixed on $M \cup \partial M$, its extension to $R^{n}$ is not unique. As a matter of fact, for every integer $m \geqq 0$, the polynomial $Q(x)=\left(\sum x_{j}\right)^{m} P(x)$ is homogeneous and agrees with $P$ on $M \cup \partial M$. However, since the partial derivatives $\partial P / \partial x_{i}$ and $\partial Q / \partial x_{i}$ differ, the transformation $\mathscr{T}_{P}$ and $\mathscr{T}_{Q}$ generated by $P$ and $Q$ differ. A direct computation shows that the transformation generated by $Q$ is given by

$$
\mathscr{T}_{Q}=\frac{m}{d+m} I+\frac{d}{d+m} \mathscr{T}_{P}
$$

where $I$ denotes the identity and $d$ is the degree of $P$. Now (16) is valid for every integer $m \geqq 0$, and if we set $t=d(d+m)^{-1}$, (16) becomes

$$
\mathscr{T}_{Q}=t \mathscr{T}_{P}+(1-t) I .
$$

By using the fact that the transformation $\mathscr{T}_{P}$ is a growth transformation, cf. [1], we can give a partial proof of (8). Indeed,

$$
P(x)=Q(x) \leqq Q\left(\mathscr{T}_{Q}(x)\right)=P\left(\mathscr{T}_{Q}(x)\right)=P\left(t \mathscr{T}_{P}(x)+(1-t) x\right)
$$

provided $t=d(d+m)^{-1}$ where $m$ is an integer. 
It is also interesting to note that when $m$ is large, the transformation $\mathscr{T}_{Q}$ is a local homeomorphism since the jacobian is (approximately) $m(d+m)^{-1}$. In fact, it is not hard to show that $\mathscr{T}_{Q}$ is a homeomorphism. This suggests that one may be able to use the topological-dynamical theory of discrete flows in order to study the asymptotic behavior of $\mathscr{T}_{Q}^{n}(x)$ when there are nonisolated singularities for $P$, or $Q$.

In the general case, where

$$
M \cup \partial M=\left\{x=\left(x_{i j}\right): x_{i j} \geqq 0, \sum_{j=1}^{q_{i}} x_{i j}=1, i=1, \cdots, k\right\}
$$

the same method yields an improvement of Theorem 6 . We are able to conclude not only that

$$
P\left(x_{i j}\right) \leqq P\left\{(1-t)\left(x_{i j}\right)+t \mathscr{T}_{P}\left(x_{i j}\right)_{i j}\right\}, \quad 0 \leqq t \leqq 1
$$

but also that

$$
\begin{aligned}
P\left(x_{i j}\right) & \leqq P\left\{\left(1-t_{i}\right)\left(x_{i j}\right)+t_{i} \mathscr{T}_{P}\left(x_{i j}\right)_{i j}\right\}, \\
0 & \leqq t_{i} \leqq 1, \quad i=1, \cdots, k,
\end{aligned}
$$

that is, not only is the number $P(x)$ smaller than (or equal to) the value of $P$ at any point along the line joining $(x)$ to $\mathscr{T}_{P}(x)$, but $P(x)$ is also less than (or equal to) the value of $P$ at any point of the $k$ dimensional rectangle determined by $(x)$ and $\mathscr{T}_{P}(x)$.

The proof of this fact is obtained by applying the original inequality [1] to the polynomials

$$
Q\left(x_{i j}\right)=\prod_{i=1}^{k}\left(\sum_{j=1}^{q_{i}} x_{i j}\right)^{n_{i}} P\left(x_{i j}\right)
$$

for integers $n_{i}$ while noting that $P$ and $Q$ agree on $M \cup \partial M$.

Another interesting consequence of this observation is that we can define proper growth transformations for nonhomogeneous polynomials with positive coefficients.

THEOREM 8. Let $P$ be a polynomial in the variables $\left(x_{i j}\right)$ with positive coefficients. Then $P$ agrees with a homogeneous polynomial $Q$ with positive coefficients on the manifold $M \cup \partial M$ and the transformation $\mathscr{T}_{Q}$ is a proper growth transformation for $P$, in fact

$$
P(x) \leqq P\left(t \mathscr{T}_{Q}(x)+(1-t) x\right), \quad(0<t \leqq 1, \quad x \in M),
$$

where equality holds if and only if $\mathscr{T}_{Q}(x)=x$.

Proof. Write $P$ in the form

$$
P=H_{0}+H_{1}+\cdots+H_{d}
$$


where $H_{l}$ is a homogeneous polynomial of degree $l$. Let

$$
Q=H_{0}\left(\sum_{j} x_{i j}\right)^{d}+H_{1}\left(\sum_{j} x_{i j}\right)^{d-1}+\cdots+H_{d},
$$

then $Q=P$ on $M \cup \partial M$. The remainder of the theorem follows from Theorem 6. Observe that $Q$ is not unique since there is great freedom in choosing the multipliers of the $H_{l}$.

Finally, the method of proof of this section can be used to extend the basic inequality (10) for homogeneous polynomials with positive coefficients to all polynomials with positive coefficients. We are grateful to Oscar Rothaus for this observation.

Theorem 9. Let $P$ be a polynomial in the variables

$$
\left\{\left(x_{i j}\right): x_{i j} \geqq 0 \sum_{j=1}^{q} x_{i j}=1, \quad i=1, \cdots, x\right\}
$$

with nonnegative coefficients. Let

$$
\mathscr{T}_{P}(x)_{i j}=x_{i j} \frac{\partial P}{\partial x_{i j}}\left[\sum_{k=1}^{q_{i}} x_{i k} \frac{\partial P}{\partial x_{i k}}\right]^{-1}
$$

Then

$$
P(x) \leqq P\left((1-t) x+t \mathscr{T}_{P}(x)\right), \quad(0<t \leqq 1) .
$$

Furthermore, equality holds if and only if $\mathscr{T}_{P}(x)=x$.

Proof. We write $P(x)$ in the form $P(x)=\sum_{l=0}^{d} H_{l}(x)$ where $H_{l}$ is a homogeneous polynomial of degree $d$. Now we introduce some dummy variables $y_{1}, y_{2}$ and enlarge the domain $M \cup \partial M$. That is, let $N \cup \partial N$ be the domain

$$
\left\{\left(x_{i j}, y_{1}, y_{2}\right):\left(x_{i j}\right) \in M \cup \partial M, y_{1} \geqq 0, y_{2} \geqq 0, y_{1}+y_{2}=1\right\}
$$

and consider the polynomial

$$
Q\left(x, y_{1}, y_{2}\right)=\sum_{l=0}^{d} H_{l}(x)\left(y_{1}+y_{2}\right)^{d-l} .
$$

$Q$ is a homogeneous polynomial with nonnegative coefficients and with $y_{1}$ and $y_{2}$ fixed, $Q$ agrees with $P$ on $M \cup \partial M$. Furthermore, we can apply Theorem 6 of this paper to $Q$. Since

$$
\begin{aligned}
\mathscr{T}_{Q}: x_{i j} \longrightarrow x_{i j} \frac{\partial Q}{\partial x_{i j}}\left[\sum_{k=1}^{q_{i}} x_{i k} \frac{\partial Q}{\partial x_{i k}}\right]^{-1} \\
=x_{i j} \frac{\partial P}{\partial x_{i j}}\left[\sum_{k=1}^{q_{i}} x_{i k} \frac{\partial P}{\partial x_{i j}}\right]^{-1}
\end{aligned}
$$


and

$$
\mathscr{T}_{Q}: y_{k} \longrightarrow y_{k} \frac{\partial Q}{\partial y_{k}}\left[\sum_{l=1}^{2} y_{l} \frac{\partial Q}{\partial y_{l}}\right]^{-1}=y_{k}
$$

it follows that $y_{1}$ and $y_{2}$ are fixed. Hence

$$
\begin{aligned}
P(x) & =Q\left(x, y_{1}, y_{2}\right) \leqq Q\left(t \mathscr{T}_{Q}\left(x, y_{1}, y_{2}\right)+(1-t)\left(x, y_{1}, y_{2}\right)\right) \\
& =Q\left(t \mathscr{T}_{P}(x)+(1-t) x, y_{1}, y_{2}\right)=P\left(t \mathscr{T}_{P}(x)+(1-t) x\right) .
\end{aligned}
$$

Furthermore, strict inequality holds for $0<t \leqq 1$ unless

$$
\mathscr{T}_{Q}\left(x, y_{1}, y_{2}\right)=\left(x, y_{1}, y_{2}\right) \text {, }
$$

that is, unless $\mathscr{T}_{P}(x)=x$.

6. Proof of Theorem 7. For clarity we consider first the special case where there is only one restraint equation. Let

$$
M \cup \partial M=\left\{x=\left(x_{j}\right): x_{j} \geqq 0 \quad \text { and } \quad \sum_{j=1}^{n} x_{j}=1\right\}
$$

Let $P_{j}=\partial P / \partial x_{j}$. Then

$$
\mathscr{T}(x)_{j}=\frac{x_{j} P_{j}}{\sum_{k} x_{k} P_{k}}=\frac{x_{j} P_{j}}{d P},
$$

is $C^{\infty}$ on the subset of $M \cup \hat{o} M$ where $P \neq 0$, and in particular is continuous on $M \cup \partial M$ at any point where $P \neq 0$ including points on the boundary $\partial M$. Therefore, $\mathscr{T}(x)$ is well defined on any local maximum of $P$ with respect to $M \cup \partial M$ whether this local maximum is on $\partial M$ or not, since $P \neq 0$ at a local maximum.

Since $P$ has positive coefficients it has no zero in $M$ unless $P \equiv 0$. On the interior of the boundary $\partial M, P$ can vanish only if $P$ is of the form $P=x_{\jmath}^{l} Q$ where $Q$ is homogeneous of degree $d-l$ and does not have $x_{j}$ as a factor. Hence $Q \neq 0$ in the interior of the face $x_{j}=0$. In this case $P \equiv 0$ on the face $x_{j}=0$ and $\mathscr{T}$ is continuously extended to the interior of the face $x_{j}=0$ by

$$
\begin{aligned}
\mathscr{T}(x)_{i} & =\frac{x_{i} Q_{i}}{d Q}, & & i \neq j \\
& =l / d, & & i=j .
\end{aligned}
$$

However, $P$ can vanish on $\partial N_{i j}$ without having a common factor and therefore $\mathscr{T}$ cannot generally be extended to $\partial N_{i j}$. For example, on the domain

$$
M \cup \partial M=\{(x, y, z): x+y+z=1, x, y, z \geqq 0\}
$$

the function $P(x, y, z)=z^{2}+4 x y$ vanishes at $z=x=0, y=1$ although 
$P$ has no common factor and in fact $\mathscr{T}_{P}$ cannot be continuously extended to $z=0, x=0, y=1$. (See the example of $\S 4$.)

More generally, the transformation $\mathscr{T}$ :

$$
\mathscr{G}^{-}(x)_{i j}=x_{i j} P_{i j}\left[\sum_{k} x_{i k} P_{i k}\right]^{-1}
$$

(where $\left.P_{i j}=\partial P / \partial x_{i j}\right)$ on the domain

$$
M \cup \partial M=\left\{\left(x_{i j}\right): x_{i j} \geqq 0, \sum_{j=1}^{q_{i}} x_{i j}=1, i=1, \cdots, k\right\}
$$

is well defined and $C^{\infty}$ on the set where no denominator $\Sigma_{k k} x_{i k} P_{i k}$ vanishes. In particular, this is true in the interior $M$ if we assume $P$ depends on at least one of each set

$$
S_{i}=\left\{x_{i j}: j=1 \cdots q_{i}\right\} i=1,2, \cdots, k .
$$

(If no term $x_{i_{0} j}, j=1 \cdots q_{i_{0}}$ appears in $P$ then we consider $\mathscr{T}_{P}$ is acting on the reduced domain without the factor

$$
\left.\left\{x_{i_{0} j} \geqq 0, \sum_{j=1}^{q_{i_{0}}} x_{i_{0} j}=1\right\} .\right)
$$

Now $\partial M=\bigcup_{i_{0}, j_{0}} N_{i_{0} i_{0}} \cup \partial N_{i_{0}, j_{0}}$, where

$$
N_{i_{0} j_{0}}=\left\{\left(x_{i j}\right): x_{i_{0} j_{0}}=0, x_{i j} \neq 0 \text { for }\langle i, j\rangle \neq\left\langle i_{0}, j_{0}\right\rangle\right\} \text {. }
$$

Let $R_{i_{1}}$ be the sum of all terms of $P$ that do not involve any factor $x_{i_{1} j}, j=1, \cdots, q_{i_{1}}$. Then for any $\left\langle i_{0}, j_{0}\right\rangle$ including $i_{0}=i_{1}, P$ can be expressed as

$$
P=x_{i_{0} j_{0}}^{l} Q(x)+R_{i_{1}}
$$

where $Q$ does not have a common factor $x_{i_{0} j_{0}}$, and hence does not vanish on $N_{i_{0} j_{0}}$. Using this decomposition one can show that $\mathscr{T}$ can be continuously extended to $N_{i_{0} j_{0}}$ as follows:

If $\Sigma_{k} x_{i k} P \neq 0$ on $N_{i_{0} j_{0}}$, then

$$
\mathscr{T}^{-}: x_{i j} \longrightarrow x_{i j} P_{i j}\left[\Sigma_{k} x_{i k} P_{i k}\right]^{-1} \text {. }
$$

If $\Sigma_{i} x_{i k} P_{i k}=0$ on $N_{i_{i} j_{0}}$ we consider two cases, $i \neq i_{0}$ and $i=i_{0}$.

(a) If $i \neq i_{0}$, then $\sum_{k k} x_{i k} Q_{i k} \neq 0$ and we define

$$
\mathscr{T}: x_{i j} \longrightarrow x_{i j} Q_{i j}\left[\Sigma_{k} x_{i k} Q_{i k}\right]^{-1} \text {; }
$$

(b) If $i=i_{0}$, and $\Sigma_{k} x_{i k} Q_{i k} \neq 0$ we define

$$
\mathscr{S}^{-}: x_{i j} \longrightarrow x_{i j} Q_{i j}\left[\Sigma_{k} x_{i k} Q_{i k}\right]^{-1} \text {. }
$$

(c) If $i=i_{0}$, and $\Sigma_{k} x_{i k} Q_{i k}=0$, we define 


$$
\mathscr{T}: x_{i_{i} j} \longrightarrow 0, j \neq j_{0} \text {, and } \mathscr{T}: x_{i_{0} j_{0}} \longrightarrow 1 \text {. }
$$

At a local maximum on $\bigcup_{i j} \partial N_{i j}$, while $P>0$, this does not obviously imply that $\sum_{j=1}^{q_{i}} x_{i j} \partial P / \partial x_{i j} \neq 0$ for each $i$. Hence a proof that $\mathscr{T}$ can be extended to be continuous at a local maximum of $P$ is most easily made by a route other than that used for the special case where there is a single restraint equation. We proceed as follows: Assume that all variables $x_{i j}$ appear in $P$. (Otherwise the discussion proceeds in a reduced space.) Then $\mathscr{T}_{P}$ maps $M$ into $M$, and for any point $x=\left(x_{i j}\right)$ in $M$, the value of $P$ at $x$ is smaller than (or equal to) the value of $P$ at any point along the line joining $x$ to $\mathscr{T}(x)$. If $x^{0}$ is an isolated local maximum of $P$ in $M \cup \partial M$, whether on the boundary $\partial M$ or not, then for all sufficiently small $\varepsilon>0$, the transformation $\mathscr{T}$ maps the connected component of

$$
\left\{(x) \mid P(x)>P\left(x^{0}\right)-\varepsilon\right\} \cap M
$$

surrounding $x^{0}$ into itself. Since these sets form a base for the neighborhoods about $x^{0}, \mathscr{T}$ can be extended to be continuous at $\left(x^{0}\right)$ by defining $\mathscr{T}\left(x^{0}\right)=x^{0}$.

Part $\mathrm{C}$ of Theorem 7 follows from the known inequalities for points in $M$ and standard limiting arguments.

Corollaries 1 and 2 to Theorem 7 are now direct applications of the above arguments.

We are grateful to the referee for some helpful comments and suggestions.

\section{REFERENCES}

1. L. E. Baum, and J. A. Eagon, An inequality with applications to statistical prediction for functions of Markov processes and to a model for ecology, Bull. Amer. Math. Soc. 73 (1967), 360-363.

2. G. R. Blakley, Homogeneous non-negative symmetric quadratic transformations, Bull. Amer. Math. Soc. 70 (1964), 712-715.

3. G. H. Hardy, J. E. Littlewood, and G. Polya, Inequalities, Cambridge University Press, 1959.

4. F. W. Wilson, The structure of the level surfaces of a Lyapunov function, J. Differential Equations 3 (1967), 323-329.

Received May 18, 1967. This work was done while the second author was at the Institute for Defense Analyses on leave from the University of Minnesota.

Institute for Defense ANAlyses

PRINCETON, NEW JERSEY 



\section{PACIFIC JOURNAL OF MATHEMATICS}

\section{EDITORS}

\section{H. ROYDEN}

Stanford University

Stanford, California

\author{
R. R. Phelps \\ University of Washington \\ Seattle, Washington 98105
}

\section{J. DugundJI}

Department of Mathematics University of Southern California Los Angeles, California 90007

\section{RICHARD ARENS}

University of California

Los Angeles, California 90024

\section{ASSOCIATE EDITORS}

\section{E. F. BECKENBACH}

B. H. NEUMANN

F. WOLF

K. YOSIDA

\section{SUPPORTING INSTITUTIONS}

UNIVERSITY OF BRITISH COLUMBIA CALIFORNIA INSTITUTE OF TECHNOLOGY UNIVERSITY OF CALIFORNIA MONTANA STATE UNIVERSITY UNIVERSITY OF NEVADA NEW MEXICO STATE UNIVERSITY OREGON STATE UNIVERSITY UNIVERSITY OF OREGON OSAKA UNIVERSITY UNIVERSITY OF SOUTHERN CALIFORNIA

\author{
STANFORD UNIVERSITY \\ UNIVERSITY OF TOKYO \\ UNIVERSITY OF UTAH \\ WASHINGTON STATE UNIVERSITY \\ UNIVERSITY OF WASHINGTON \\ AMERICAN MATHEMATICAL SOCIETY \\ CHEVRON RESEARCH CORPORATION \\ TRW SYSTEMS
}

NAVAL WEAPONS CENTER

Mathematical papers intended for publication in the Pacific Journal of Mathematics should be in typed form or offset-reproduced, double spaced with large margins. Underline Greek letters in red, German in green, and script in blue. The first paragraph or two must be capable of being used separately as a synopsis of the entire paper. It should not contain references to the bibliography. Manuscripts, in duplicate if possible, may be sent to any one of the four editors. All other communications to the editors should be addressed to the managing editor, Richard Arens, University of California, Los Angeles, California 90024.

Each author of each article receives 50 reprints free of charge; additional copies may be obtained at cost in multiples of 50 .

The Pacific Journal of Mathematics is published monthly. Effective with Volume 16 the price per volume (3 numbers) is $\$ 8.00$; single issues, $\$ 3.00$. Special price for current issues to individual faculty members of supporting institutions and to individual members of the American Mathematical Society: $\$ 4.00$ per volume; single issues $\$ 1.50$. Back numbers are available.

Subscriptions, orders for back numbers, and changes of address should be sent to Pacific Journal of Mathematics, 103 Highland Boulevard, Berkeley 8, California.

Printed at Kokusai Bunken Insatsusha (International Academic Printing Co., Ltd.), 7-17, Fujimi 2-chome, Chiyoda-ku, Tokyo, Japan.

PUBLISHED BY PACIFIC JOURNAL OF MATHEMATICS, A NON-PROFIT CORPORATION

The Supporting Institutions listed above contribute to the cost of publication of this Journal, but they are not owners of publishers and have no responsibility for its content or policies. 


\section{Pacific Journal of Mathematics}

\section{Vol. 27, No. $2 \quad$ February, 1968}

Leonard E. Baum and George Roger Sell, Growth transformations for

functions on manifolds ............................ 211

Henry Gilbert Bray, A note on CLT groups ................... 229

Paul Robert Chernoff, Richard Anthony Rasala and William Charles

Waterhouse, The Stone-Weierstrass theorem for valuable fields....... 233

Douglas Napier Clark, On matrices associated with generalized

interpolation problems ................................

Richard Brian Darst and Euline Irwin Green, On a Radon-Nikodym theorem for finitely additive set functions . ...................... 255

Carl Louis DeVito, A note on Eberlein's theorem..................... 261

P. H. Doyle, III and John Gilbert Hocking, Proving that wild cells exist . . . 265

Leslie C. Glaser, Uncountably many almost polyhedral wild $(k-2)$-cells in

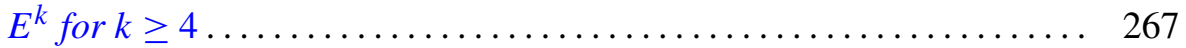

Samuel Irving Goldberg, Totally geodesic hypersurfaces of Kaehler

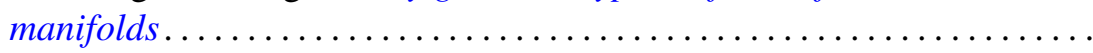

Donald Goldsmith, On the multiplicative properties of arithmetic functions .................................... 283

Jack D. Gray, Local analytic extensions of the resolvent ............ 305

Eugene Carlyle Johnsen, David Lewis Outcalt and Adil Mohamed Yaqub,

Commutativity theorems for nonassociative rings with a finite division ring homomorphic image ....................

André (Piotrowsky) De Korvin, Normal expectations in von Neumann

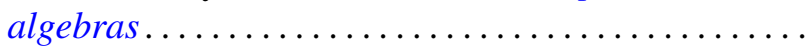

James Donald Kuelbs, A linear transformation theorem for analytic

Feynman integrals..........................

W. Kuich, Quasi-block-stochastic matrices ................... 353

Richard G. Levin, On commutative, nonpotent archimedean

semigroups ............................... 365

James R. McLaughlin, Functions represented by Rademacher series ... . . . 373

Calvin R. Putnam, Singular integrals and positive kernels............ 379

Harold G. Rutherford, II, Characterizing primes in some noncommutative

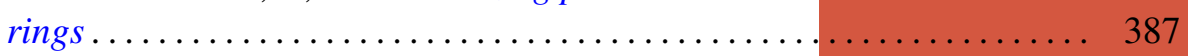

Benjamin L. Schwartz, On interchange graphs................... 393

Satish Shirali, On the Jordan structure of complex Banach *algebras . . . . . 397

Earl J. Taft, A counter-example to a fixed point conjecture............. 405

J. Roger Teller, On abelian pseudo lattice ordered groups ..... 